\title{
AVALIAÇÃO DA INTEGRIDADE DE OCUPANTES DE VEÍCULOS TIPO PICK-UP EM CASO DE CAPOTAMENTO: UMA ABORDAGEM NUMÉRICA
}

\author{
Rita C. Silva ${ }^{1}$, Maria Alzira A. Nunes ${ }^{1}$, Alessandro B. de S. Oliveira ${ }^{1}$ \\ ${ }^{1}$ Universidade de Brasília, Faculdade UnB Gama, Engenharia Automotiva \\ E-mails: ritasilva@unb.br, maanunes@unb.br, abso@unb.br
}

\section{RESUMO}

O capotamento de veículos representa uma das principais causas de lesões e morte de seus ocupantes. Este tipo de acidente pode ocorrer tanto em vias rápidas, quanto em áreas específicas como, por exemplo, interior de minas onde a velocidade dos veículos não atinge valores elevados. Diante deste cenário, a instalação de dispositivos de segurança que sejam capazes de atenuar alguns dos efeitos maléficos deste evento aos condutores e passageiros, representa uma medida viável, é o caso, por exemplo, de um dispositivo passivo denominado ROPS (Rollover Protection System). Este dispositivo pode ser construído utilizando-se barras soldadas de aço que constituem uma estrutura a ser instalada externa ou internamente (tipo gaiola) ao veículo. Tal estrutura tem por objetivo minimizar a intrusão (deformação) da estrutura veicular em caso de capotamento e, assim, preservar a integridade dos passageiros. Considerando uma abordagem numérica (simulação), o presente trabalho descreve e apresenta os principais resultados da segunda fase de um total de três, de um projeto que visa ao estudo, avaliação e o projeto estrutural de um sistema de proteção contra capotagem (ROPS). Este artigo consiste na avaliação da integridade de ocupantes em caso de capotamento de veículos tipo pick-up (caminhonete), visando principalmente aplicação em ambiente de mineração. Ressalta-se que previamente a este trabalho (denominada primeira fase) o modelo numérico em elementos finitos da caminhonete e do setup de teste foi desenvolvido. A terceira fase se presta a analisar a influência do ROPS na avaliação dos critérios de lesão dos passageiros quando do capotamento do veículo. Desta maneira a eficiência do dispositivo de segurança poderá ser avaliada considerando danos e lesões aos ocupantes do veículo. A metodologia numérica deste trabalho consiste na simulação do teste experimental de capotamento (rollover) denominado Roof Crush (regulamentação FMVSS 216) utilizando-se o pacote computacional de elementos finitos não-linear, LS-Dyna ${ }^{\circledR}$ considerando dummies no interior do veículo. Os critérios de lesões de pescoço e cabeça considerados estão descritos na regulamentação americana FMVSS 208.

\section{INTRODUÇÃO}

Dentre os acidentes de veículos citam-se as colisões frontais e laterais, além daqueles que envolvem o capotamento ou rolagem do veículo (rollover crashes); sendo que estes últimos podem ser considerados como um evento de longa duração ( 1 a 4 s em $80 \%$ dos casos). Além 
disso, os efeitos que tal tipo de acidente pode ocasionar na integridade física dos ocupantes no interior do veículo, têm despertado o interesse de vários pesquisadores da área de segurança veicular [1, 2, 3, 4]. Conforme mostrado em [4], os acidentes envolvendo capotagem do veículo respondem por um número muito grande de lesões graves dos passageiros comparativamente a outros tipos de colisões.

De fato, a cinemática que o corpo humano pode sofrer no habitáculo durante este tipo de acidente é complexa e podem ocorrer múltiplos contatos do passageiro com o interior do veículo, sobretudo com as colunas A, B e o teto. Considerando esses movimentos, percebe-se que os pontos mais afetados são a cabeça e o pescoço (cervical) [3]. Salienta-se que de forma geral, tal comportamento independe se um acidente desta natureza se dá com veículos urbanos ou com aqueles que desempenham atividades em áreas específicas (mineração, construção...).

Cabe salientar ainda, que estudos com esta perspectiva vêm sendo desenvolvidos ao longo dos anos e podem ser tanto experimentais quanto numéricos $[2,5,6,7,8]$.

O trabalho desenvolvido em [2] tem um caráter numérico e visa demonstrar o uso do software MADYMO $^{\circledR}$ na simulação da cinemática do ocupante no interior do veículo. De fato, buscase avaliar a efetividade de critérios de lesão de cabeça como o HIC (Head Injury Criteria) e o HIP (Head Impact Power)/PVP (Peak Virtual Power), em casos de acidentes ocasionados por capotamento. Diante de tal objetivo, dois casos reais de capotamento foram modelados no MADYMO®, além do teste experimental Dolly Test presente na norma SAE J2114.

Os resultados obtidos demonstraram que a simulação cinemática e a análise de lesões de cabeça via MADYMO ${ }^{\circledR}$, em caso de capotamentos, é relevante. Da comparação entre as intrusões do teto do veículo e as lesões de cabeça, os resultados das simulações do Dolly Test (SAE J2114) mostram que a velocidade de intrusão parece ser a causa principal das lesões registradas nos casos estudados. Assim, conclui-se que a avaliação numérica da dinâmica veicular e da cinemática do ocupante pode ser analisada usando-se o MADYMOß.

Parenteau, C. S. et al [5] conduziram seu estudo, primeiramente, identificando os tipos de capotamento mais usuais, bem como as circunstâncias de ocorrência, em acidentes reais. Em seguida, dentre estes se identificou o modo mais comum, ou seja, o de maior estatística, o qual teve a cinemática veicular correspondente comparada com dados disponíveis em testes de laboratório. De acordo com seu estudo, dentre as formas de capotamento analisadas, a mais comum é a denominada "trip-over" (Fig. 1) tanto em carros de passeio (57\%) quanto em veículos leves (51\%), estando os veículos do tipo pick-ups entre estes.

Neste tipo de mecanismo de capotamento, o veículo tem seu movimento lateral repentinamente reduzido ou interrompido e, em $90 \%$ dos casos isto se dá a partir do contato com algum obstáculo no solo. Dentre os resultados de testes experimentais utilizados no estudo, têm-se os referentes ao FMVSS 208 (Federal Motor Vehicle Safety Standard) dolly, ADAC corkscrew, soil-trip, curb-trip and ditch fall-over, conforme ilustrado na Fig. 2. 


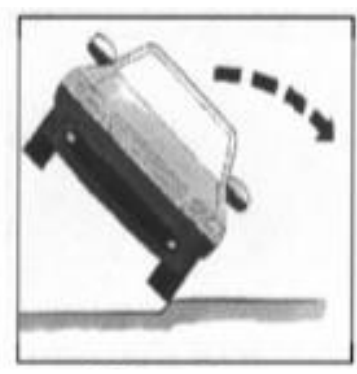

Figura 1 - Ilustração do modo de capotamento denominado "trip-over" [9].

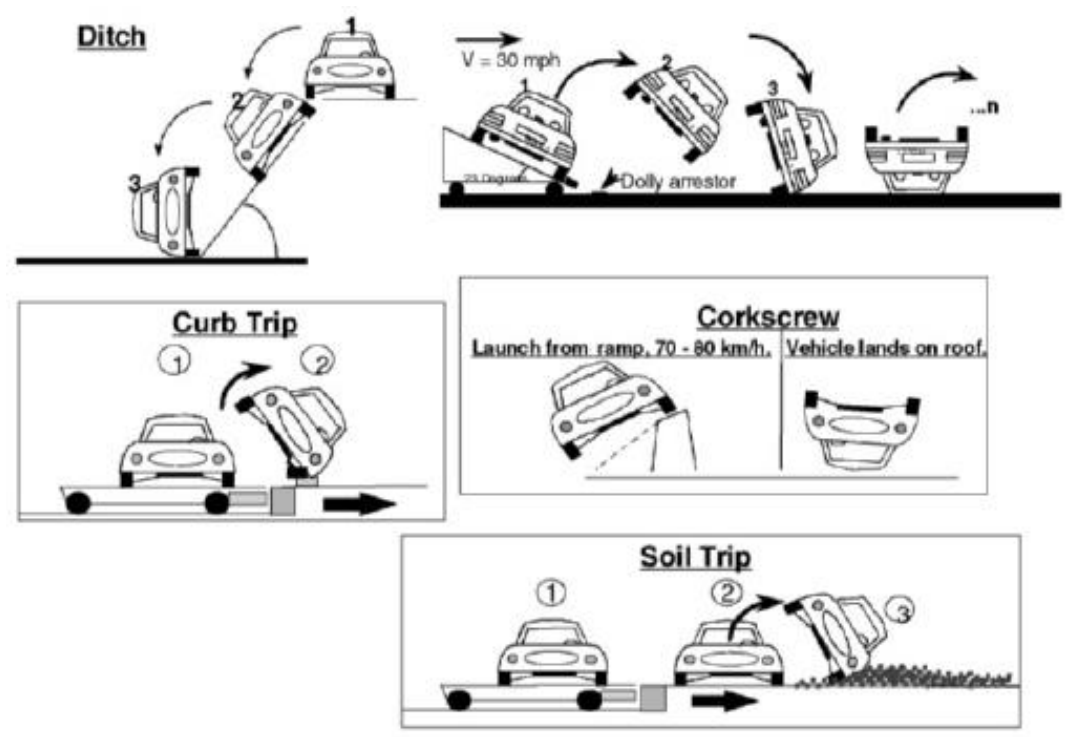

Figura 2 - Ilustração dos testes de laboratórios considerados em [6]

Os dados coletados em testes de laboratório permitiram uma maior compreensão do movimento do ocupante (cinemática), no caso o motorista com cinto de segurança, além de possibilitar a determinação das lesões que este pode sofrer durante a rolagem. Independentemente dos modos de capotamento (Fig. 2), as lesões mais comuns afetavam a cabeça e o tórax.

Kerrigan, J. R. et al [6] em seu estudo, visou verificar a reprodutibilidade de um ensaio experimental de capotamento levando em consideração as condições do teste, do veículo e a resposta do ocupante. Oito testes de rolagem foram realizados utilizando três veículos Ford Explorer 2002, sendo 5 testes não-destrutivos com baixa velocidade (Low Speed: 19,3 km/h) e 3 destrutivos sob alta velocidade (High Speed: 48,3 km/h). Cada um dos veículos foi instrumentado com 24 sensores e ensaiado com o dispositivo de teste "Deceleration rollover sled method". Os resultados mostraram que o aparato de teste utilizado permite repetitividade, ou seja, as respostas tanto em termos de intrusão do veículo, como com relação às verificações dos critérios de lesão nos dummies podem ser reproduzidas, mas estes mesmos resultados se mostraram sensíveis às condições de teste.

Stephenson, R. R. [7] afirma que o teste dinâmico de capotagem é uma das metodologias a serem seguidas, a fim de reduzir o número de vítimas fatais e lesões graves ocasionadas pelo evento de capotamento. Em seu trabalho, discute-se a eficiência dos três testes dinâmicos mais utilizados no estudo de rolagem de veículos: FMVSS 208 "Rollover Dolly", o 
"Controlled Rollover Impact System" (CRIS) e o "Jordan Rollover System" (JRS). Após análise, o autor conclui que o teste de melhor repetitividade é o JRS.

O autor também afirma que a pesquisa deve avançar neste sentido, para que haja concordância nas condições iniciais, a fim de se propor implementações no dummy antropomórfico (instrumentação), assim como nos critérios de lesões referentes ao pescoço e tórax utilizados pela FMVSS e a NCAP (New Car Assessment Program).

Ainda visando à verificação da resposta cinemática do ocupante do veículo, durante um evento de capotamento, Yan, L. et al [8] compararam os resultados obtidos, referente a esta avaliação, de dois testes experimentais modelados numericamente. Para tanto dois softwares foram utilizados LS-Dyna ${ }^{\circledR}$ e o MADYMO ${ }^{\circledR}$, o modelo do veículo em Elementos Finitos (FE) é de um FORD Explorer 2003 desenvolvido pela National Crash Analysis Centre (NCAC).

O modelo utilizado foi validado para colisão frontal, mas também para o teste experimental segundo a FMVSS 216 considerando dois ângulos distintos de pitch e roll. Os modelos numéricos em elementos finitos para simulação virtual dos testes experimentais do JRS e do Over the Road Rollover (OTRR) foram construídos e a resposta dos passageiros ao evento de capotamento avaliada nas duas configurações comparadas.

Mediante o exposto acima, percebe-se que muito se tem feito de modo a compreender a cinemática e a dinâmica de passageiros e veículos em eventos de capotamento. Para tanto, trabalhos de cunho experimental ou numérico visam a analisar o comportamento do veículo durante eventos desta natureza e, além disso, como tal afeta a integridade dos passageiros.

Neste sentido, o presente trabalho vem contribuir apresentando a simulação numérica de um teste experimental de rollover denominado Roof Crush (FMVSS 216) de um veículo tipo pick-up (caminhonete), os quais são utilizados em ambientes de mineração e estão susceptíveis ao fenômeno da rolagem/capotamento.

Assim, após desenvolvimento do modelo numérico em elementos finitos não-linear utilizando o software comercial LS-Dyna ${ }^{\circledR}$ (primeira fase), esta etapa da pesquisa, descrita neste trabalho, foca na simulação do teste Roof Crush considerando quatro dummies do tipo Híbrido III 50\%, dois frontais e dois traseiros, tendo como meta principal verificar a integridade destes manequins segundo critérios de lesões estabelecidos na regulamentação americana FMVSS 208.

Como será discutido no item 1, a escolha pela simulação numérica do ensaio experimental Roof Crush se deu, principalmente, visando minimizar critérios relativos ao tempo e custo de processamento computacional.

Ressalta-se que o escopo do trabalho completo é mais abrangente e ao fim se reside na proposição de um dispositivo de segurança externo denominado ROPS (Rollover Protection System) a ser aplicado em pick-ups com a finalidade de assegurar a integridade dos ocupantes em ambientes de mineração.

\section{CONSIDERAÇÕES ACERCA DO MODELO NUMÉRICO DA PICK-UP E MODELO NUMÉRICO DO TESTE ROOF CRUSH}


Este item se propõe a apresentar, de forma sucinta, as características do modelo de pick-up utilizado, bem como os procedimentos numéricos assumidos para adaptação do modelo para fins do estudo de capotamento (rollover). Conforme dito, o trabalho aqui discutido, no que se refere à simulação numérica do fenômeno, corresponde à segunda fase em um total de três, de um projeto que visa a desenvolver um dispositivo de segurança externo denominado ROPS (RollOver Protection System) para veículos tipo 4 x 4, que trafegam interna e externamente ao ambiente de mina.

Nesta fase, o objetivo é desenvolver uma metodologia de avaliação da integridade dos ocupantes de veículo, a partir da simulação numérica do teste experimental Roofcrush (FMVSS 216) [10]. É nesse sentido, que a presente seção se faz necessária, visto que a modelagem e as premissas/considerações de simulação foram feitas na primeira fase do estudo. No entanto, tais considerações serão apenas mencionadas, visto que o objetivo principal do trabalho é verificar os critérios de lesões de cabeça e pescoço.

\subsection{Modelo em Elementos Finitos da Pick-up}

O modelo em elementos finitos da pick-up utilizada no presente estudo encontra-se disponível ao público no site da National Crash Analysis Center (NCAC) (http://www.ncac.gwu.edu/vml/models.html), resultado de um trabalho conjunto entre a Federal Highway Administration (FHWA), National Highway Traffic Safety Administration (NHTSA) e a George Washington University (GWU). Trata-se de uma pick-up da Chevrolet modelo Silverado 2007, quatro portas, cabine dupla, motor 4.8 L V8, transmissão automática, pneus tipo P245/70R17, distância entre os eixos de 3,664 m e posição do CG a 1,664 m, a partir das rodas dianteiras.

De fato, a NCAC e a GWU desenvolvem modelos não lineares em elementos finitos, para serem utilizados pelo software LS-Dyna®. A aplicação visa às pesquisas voltadas para a área de segurança em transportes. Normalmente, estes modelos contam com mais de 1 milhão de elementos (excluindo os componentes do interior do veículo: banco, revestimento interno, cintos, consoles, etc.).

O modelo FE Silverado da NCAC foi validado para impactos frontais de acordo com a New Car Assessment Programme (NCAP) tendo uma massa de $2.617 \mathrm{~kg}$. Os elementos que compõem a malha de elementos finitos do veículo são mostrados na Tab. 1, com tamanho médio de $0,011 \mathrm{~m}$. 
Tabela 1 - Tipos de elementos presentes no modelo da pick-up

\begin{tabular}{l|l}
\hline Elementos & Quantidade \\
\hline Elemento Shell & 873144 \\
\hline Elemento Solid & 53293 \\
\hline Elemento Beam & 2665 \\
\hline Elemento Discrete & 32 \\
\hline Elemento Mass & 321 \\
\hline $\begin{array}{l}\text { Elemento Seatbelt } \\
\text { Accelerometer }\end{array}$ & 4 \\
\hline Airbag & 4 \\
\hline Soldas de ponto & 6903 \\
\hline Junta esférica & 9 \\
\hline Junta revoluta & 30 \\
\hline Total & $\mathbf{9 3 7 . 0 7 3}$ \\
\hline
\end{tabular}

Os elementos do tipo Shell são TRIA e QUAD com três e quatro nós. Os elementos sólidos são definidos como PENTA e HEXA com seis e oito nós.

A validação do modelo da NCAC usando LS-dyna ${ }^{\circledR}$ consome 16 CPU's em um total de $16 \mathrm{~h}$ para $150 \mathrm{~ms}$ de simulação. Baseado nesta informação, se for considerado que um capotamento dura em torno $1000 \mathrm{~ms}$ têm-se $107 \mathrm{~h}$ de processamento. Isto indica que o modelo precisa ser simplificado para que seja viável.

Desta forma, duas implementações foram realizadas: aumentou-se o tamanho dos elementos e algumas partes do modelo foram tratadas como corpos rígidos. Assim, os elementos reduziram de 937.073 para 153.616 . Isto porque o time step passou de 1E-6 s (condição inicial) para 2,5E-6 s. O tamanho dos elementos também se modificou de $11 \mathrm{~mm}$ para $28 \mathrm{~mm}$, assim o número de nós reduziu de 942.677 para 160.057.

A Figura 3 ilustra o veículo dividido em parte rígida (chassi, transmissão e motor) e parte deformável. 


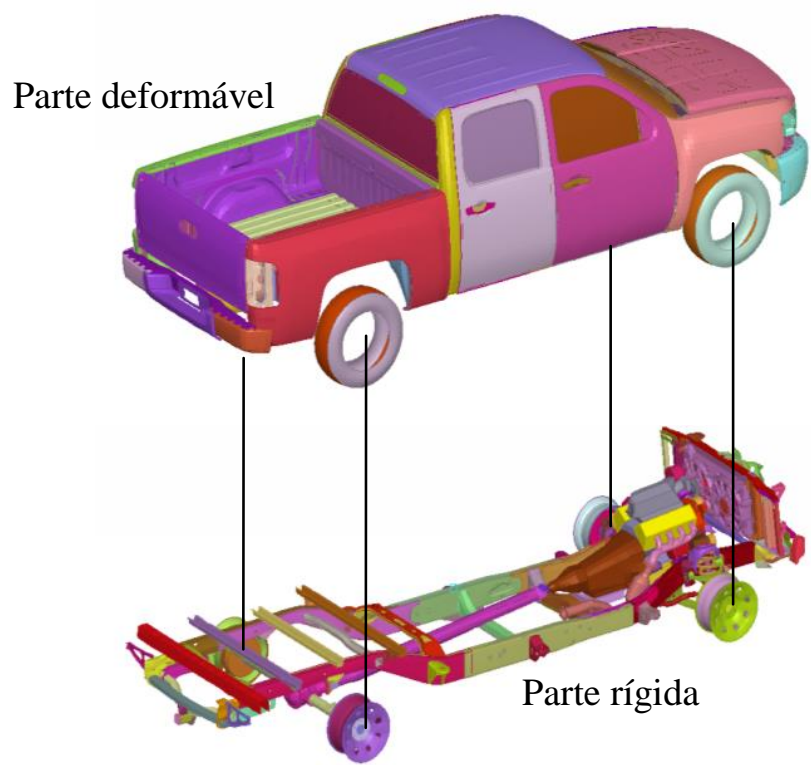

Figura 3 - Modelo em elementos finitos da pick-up

Silverado: partes deformáveis e partes rígidas

\section{2 - Considerações quanto à modelagem numérica em elementos finitos do teste experimental Roof Crush}

De posse de um modelo numérico mais otimizado da pick-up, passou-se para a validação do modelo numérico não linear em elementos finitos do teste Roofcrush [10] usando o LS-Dyna ${ }^{\circledR}$. Para tanto, utilizou-se um modelo numérico auxiliar referente ao teste experimental Dolly test rollover [9]. De fato, a partir da simulação numérica deste teste definiram-se a área de impacto e os dados de entrada para a simulação do Roofcrush.

Inúmeras simulações do Dolly rollover foram realizadas visando obter uma área de impacto desejada, a qual é localizada na lateral do teto do veículo. Para obtenção desta área de impacto foram realizados ajustes a cada simulação nos seguintes parâmetros: definição do tipo de contato entre a pickup e o dispositivo dolly (contato tipo pure master-slave); formato e número de elementos usados em ambas as superfícies de contato; atrito na interface de contato; características dos pneus tais como material, pressão, tamanho, rigidez, etc.; e por fim as características da suspensão, incluindo sua massa e propriedades de inércia. $\mathrm{O}$ objetivo deste ajuste é obter com o teste Dolly rollover a mesma área de impacto definida pelo teste Roof Crush. Após o refinamento da malha da pickup e os ajustes citado anteriormente, considerando o evento de rolagem com duração de 1 $\mathrm{s}$, tem-se que o tempo de processamento passou a $21 \mathrm{~h}$ com um número de 4 CPU's. Percebe-se que nesta condição, ainda não houve a inserção dos dummies o que penalizaria ainda mais os aspectos de número de CPU's e tempo de processamento. A Figura 4 ilustra o aspecto resultante da área de impacto no veículo (primeiro contato com o solo) simulando o teste Dolly rollover. 

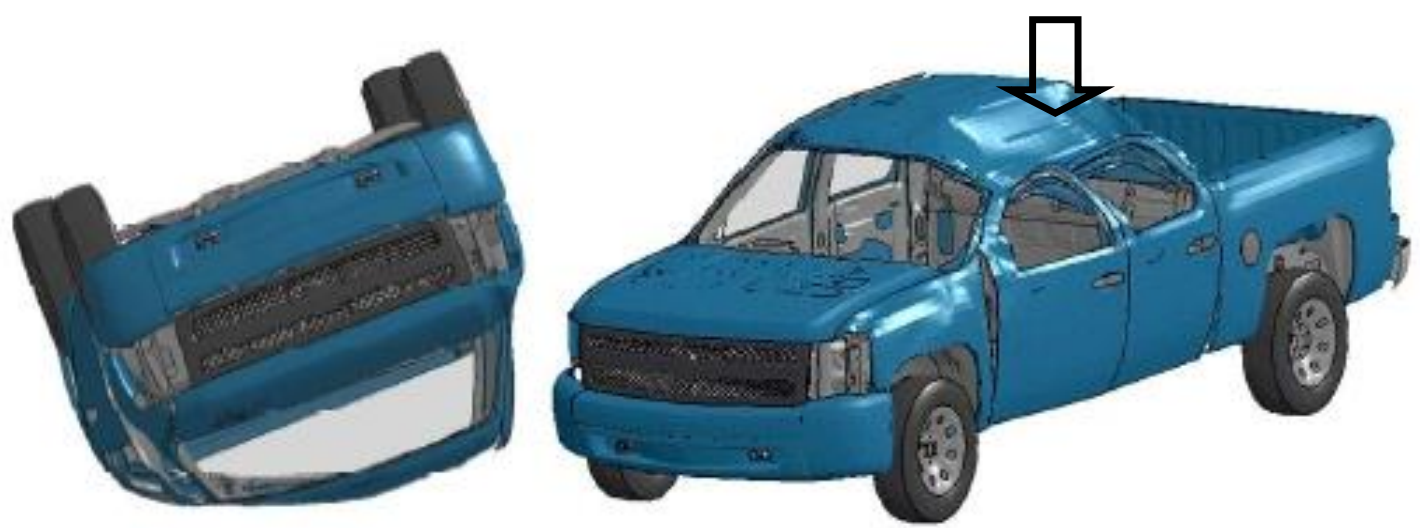

Figura 4 - Área de impacto no veículo após conclusão do processo de simulação do teste experimental Dolly rollover

Outras duas saídas são de interesse no processo final de simulação do Dolly rollover, são elas: a energia interna de deformação responsável pela intrusão do teto do veículo e a velocidade de translação do CG.

Isto porque para a simulação numérica do Roofcrush é necessário definir a massa da placa de aplicação do carregamento para que a massa associada seja equivalente à energia que provoca a intrusão segundo o Dolly teste, assim como a velocidade do CG no momento do primeiro impacto. Desta forma, a mesma é estimada em 1,5 ton. Este dado é obtido considerando-se uma energia cinética de 27E+6 N.mm e uma velocidade resultante no CG do veículo de $6000 \mathrm{~mm} / \mathrm{s}$, ou seja, $m=\frac{2 \cdot E_{C}}{v^{2}}=1,5 t$.

$\mathrm{O}$ valor da força correspondente à energia acima explicitada é, no instante $\mathrm{t}=0,7$ $\mathrm{s}$, igual a $\mathrm{F} \approx 119.000 \mathrm{~N}$. Esta foi obtida por intermédio da simulação numérica do dispositivo Dolly teste, sendo estimada a partir do gráfico da Fig. 5, no instante de tempo em que termina o primeiro impacto.

Acrescenta-se ainda que o que prescreve a norma FMVSS 216 serviu apenas de referência para a geometria do dispositivo, Fig.5. A adaptação se encontra no fato de se usar a energia vinda de um teste dinâmico Dolly rollover em um teste de características quasi-estática. Isto porque se deseja verificar a integridade dos ocupantes, em caso do impacto do veículo contra o solo considerando-se uma força desta magnitude. 


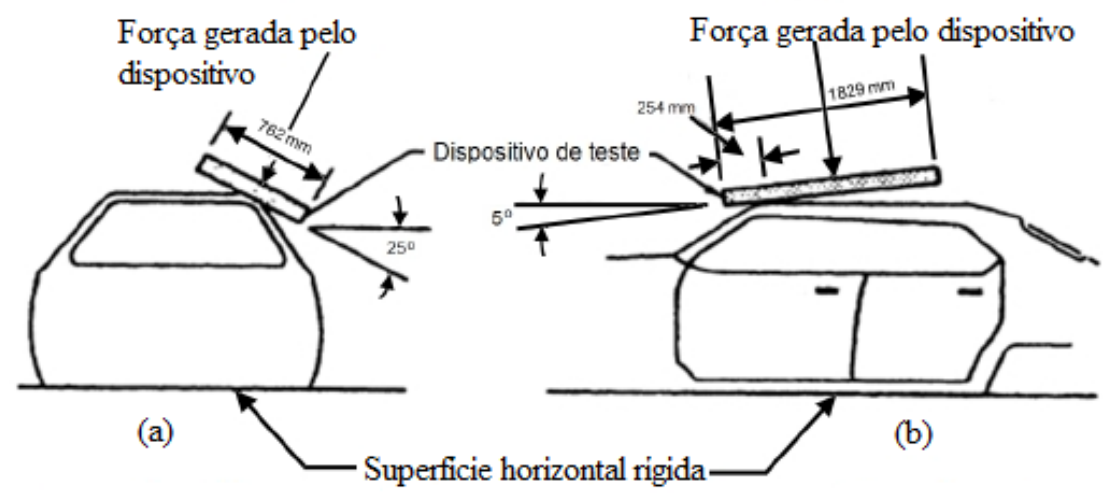

Figura 5 - Esquema de aplicação da força gerada pelo dispositivo de teste. (a) vista frontal (b) vista lateral - Fonte: FVMSS 216.

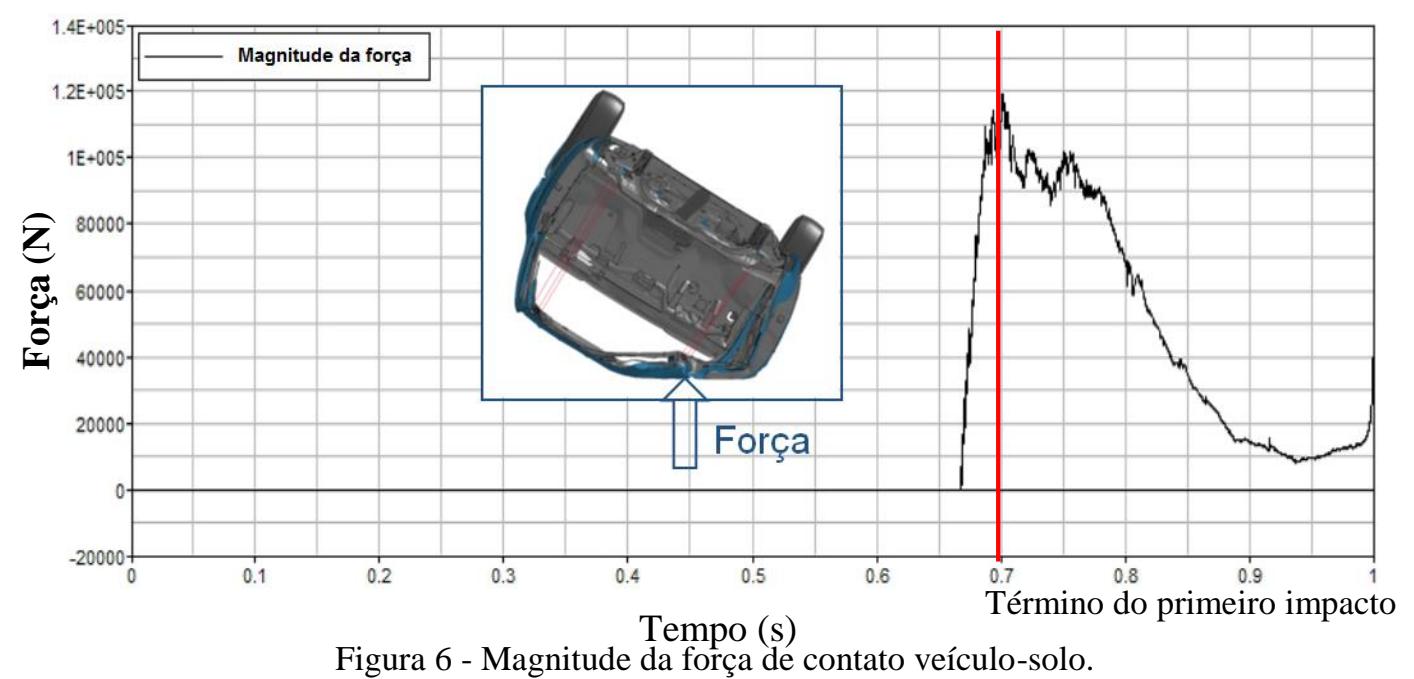

\section{2 - INTEGRIDADE DOS OCUPANTES: MECANISMOS E CRITÉRIOS DE LESÃO}

Este tópico tratará dos critérios envolvidos no que se refere a danos e lesões que envolvem ocupantes de veículos em caso de acidentes. Os valores estão baseados na norma FMVSS 208.

A lesão está relacionada a uma resposta biomecânica alterada, ou seja, partes do corpo são solicitadas além de sua capacidade e podem se deformar além do que é reparável. Tal resulta em um dano permanente na estrutura anatômica ou altera o desempenho normal de determinadas funções. A causa principal destas lesões são carregamentos não usuais sobre o corpo humano, em especial os de impacto.

No presente trabalho, são consideradas duas regiões do corpo: a cabeça e o pescoço. Os critérios a serem utilizados são, em termos da cabeça, HIC (Head Injury Criteria) usado para medir o potencial de lesão na cabeça (cérebro) oriundo da exposição a acelerações lineares, além da aceleração máxima de pico admitida para um intervalo pré-definido (A3ms). Este último critério encontra-se na antiga norma FMSSA 208. 
No caso do pescoço, o critério de lesão considera o cálculo do parâmetro $N_{i j}$, que, conforme as condições de carregamento, pode ter quatro (4) combinações possíveis: tração-extensão, tração-flexão, compressão-extensão ou compressão-flexão. Ressalta-se que estes critérios são mais bem detalhados nos itens que se seguem.

\section{1 - Lesões associadas à cabeça: verificação dos critérios}

Conforme dito, o critério a ser utilizado é o HIC, o qual é amplamente utilizado para mensurar o risco de lesões na cabeça e, consequentemente, cérebro.

Este critério se baseia na integral da aceleração resultante $\left(a=\sqrt{a_{x}^{2}+a_{y}^{2}+a_{z}^{2}}\right)$, que representa a aceleração calculada no centro de gravidade da cabeça, expressa em unidades (múltiplo) da aceleração da gravidade $\left(\mathrm{g}=9,81 \mathrm{~m} / \mathrm{s}^{2}\right)$. Os tempos $t_{1}$ e $t_{2}$ são pontos no tempo registrados ao longo do crash e neles o HIC é máximo. A Equação 1 traz a expressão que estima o HIC.

$$
H I C=\left(t_{2}-t_{1}\right)\left[\frac{1}{\left(t_{2}-t_{1}\right)} \int_{t_{1}}^{t_{2}} a(t) d t\right]^{2,5}
$$

Os intervalos de tempo máximo considerados podem ser de $15 \mathrm{~ms}$ para acidentes que envolvem contato direto da cabeça com o interior do veículo, ou $36 \mathrm{~ms}$ em caso contrário. Originalmente, o valor limite para HIC era de 1000, independente do sexo e tamanho do manequim e, para resultados acima deste valor esperavam-se lesões graves e permanentes na cabeça.

Entretanto, este critério sofreu alterações apresentadas pela National Highway Traffic Safety Administration, 2001 (NHTSA), o que resultou na consideração de intervalo de tempo crítico de $15 \mathrm{~ms}$, sendo o mais comumente usado e uma revisão dos limites de HIC para vários tamanhos de dummies. A denominação deste critério é normalmente, HIC15 para o intervalo de $15 \mathrm{~ms}$ e HIC36 para $36 \mathrm{~ms}$. A Tabela 2 ilustra os valores que são aplicáveis. Neste trabalho, o valor de referência a ser considerado é o HIC15, correspondente ao dummy Hibrido III percentil 50\% masculino, assim $\boldsymbol{H I C 1 5}=\mathbf{7 0 0}$.

Tabela 2 - Critério de lesão na cabeça HIC segundo proposta NHTSA

\begin{tabular}{c|c|c|c|c|c}
\hline & $\begin{array}{c}\text { Híbrido III } \\
50 \% \text { Masc. }\end{array}$ & $\begin{array}{c}\text { Híbrido III } \\
50 \% \text { Fem. }\end{array}$ & $\begin{array}{c}\text { Híbrido III } \\
6 \text { anos }\end{array}$ & $\begin{array}{c}\text { Híbrido III } \\
3 \text { anos }\end{array}$ & 12 meses \\
\hline \hline HIC36 & 1000 & 1000 & 1000 & 900 & 660 \\
\hline HIC15 & 700 & 700 & 700 & 570 & 390 \\
\hline
\end{tabular}

Outro aspecto a se considerar relativamente às lesões de cabeça, diz respeito à aceleração de pico em um intervalo de tempo correspondente a $3 \mathrm{~ms}$, denominada A3ms. Assim, a resultante da aceleração no centro de gravidade da cabeça não deve exceder 80 g's ( $g$ é a aceleração da gravidade igual a $9,81 \mathrm{~m} / \mathrm{s}^{2}$ ), para um intervalo de tempo correspondente a $3 \mathrm{~ms}$. A exceção a valores maiores de aceleração é 
admitida para intervalos de tempo inferiores a $3 \mathrm{~ms}$. Assim o valor limite de A3ms é de 80 g's.

\section{2 - Lesões associadas ao pescoço: verificação dos critérios}

A definição das possíveis lesões de pescoço passa pela compreensão da forma usual de se classificar os mecanismos de trauma deste. Do ponto de vista da biomecânica e em se considerando a análise de esforços, tem-se na Fig. 7 a descrição de carregamentos no pescoço.

Percebe-se da Fig. 7 que podem ocorrer carregamentos axiais de tração e compressão; força de cisalhamento (deslizamento) no plano transversal ao pescoço; torção e flexão. Nesta última, percebe-se que as setas indicam a aplicação de um binário (forças de mesmo módulo e direção com sentido contrário). Os movimentos originários desta flexão podem ser o movimento da cabeça para frente ou o movimento da cabeça para trás, como ilustra a Fig.8.

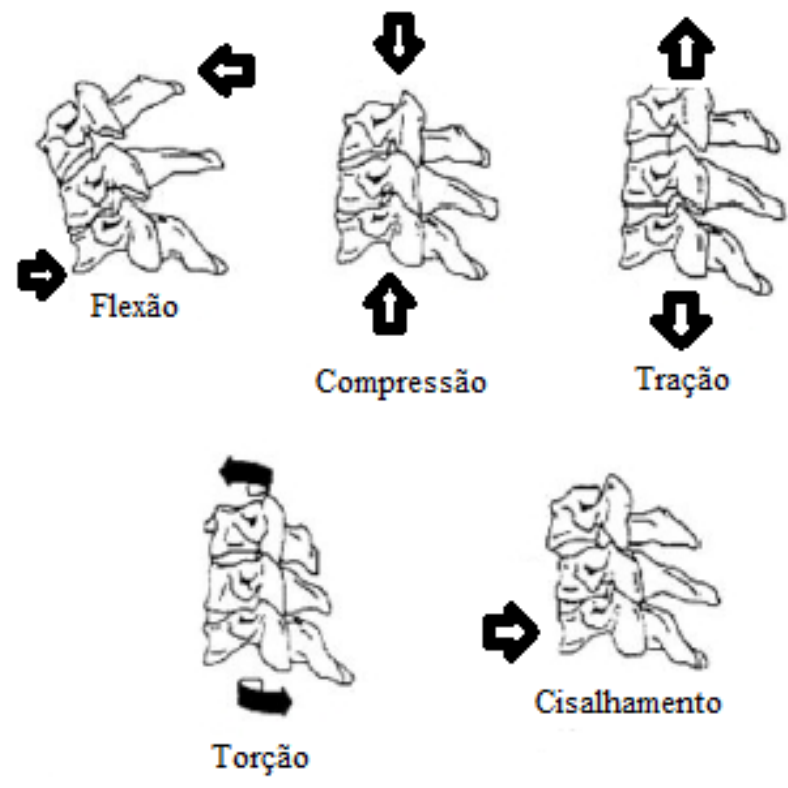

Figura 7 - Esforços possíveis atuantes no pescoço.

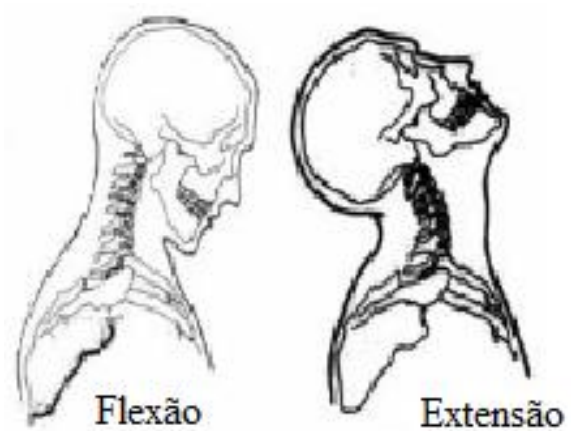

Figura 8 - Movimento da cabeça configurando um esforço de flexão. 
Considerando um sistema de referência em que o eixo " $x$ " esteja transversal ao pescoço, "z" longitudinal ao pescoço e "y" perpendicular ao plano "xz", Fig. 9 (a), têm-se que a força de cisalhamento $F_{x}$, a força axial $F_{z}$ e o momento fletor $M_{y}$ devem ser extraídos na região superior do pescoço, denominada côndilo occipital em Fig. 9 (b).
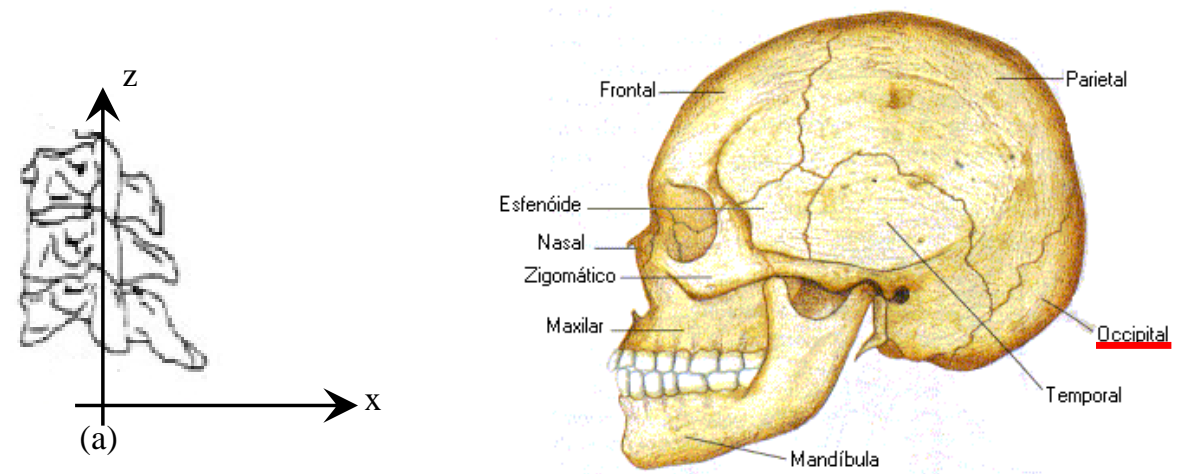

(b)

Figura 9 - Sistema de referência aplicável ao pescoço.

A Equação 2 mostra como é feito o cálculo do critério de lesão do pescoço, segundo a FMVSS 208.

$N_{i j}=\frac{F_{z}}{F_{z c}}+\frac{M_{o c y}}{M_{y c}}$

onde $\mathrm{F}_{\mathrm{zc}}$ e $\mathrm{M}_{\mathrm{yc}}$ são valores estabelecidos pela norma, segundo o tipo de esforço no pescoço. Assim, $\mathrm{F}_{\mathrm{zc}}$ igual a $6.806 \mathrm{~N}$ ou $6.160 \mathrm{~N}$, quando de tração ou compressão, respectivamente, conforme Fig. 7. $\mathbf{M}_{\mathrm{yc}}$ assume os valores de 310 N.m ou 135 N.m, no caso de momento de flexão ou extensão na região do côndilo occipital (Fig. 8 e Fig. 9 (b)), respectivamente, segundo FMVSS 208. Estes valores são estabelecidos para o dummy Híbrido III 50\%, masculino. Isto porque estes valores variam segundo idade e sexo.

Já os valores de $\mathrm{F}_{\mathrm{z}}$ e $\mathrm{M}_{\mathrm{ocy}}$ são os valores medidos durante a simulação do Roofcrush, registrados por célula de carga colocada na parte superior do pescoço (côndilo occipital). Ressalta-se que além do critério de lesão $N_{i j}$, segundo a FMVSS 208, deve-se verificar se os valores de pico são toleráveis. Segundo a norma item S 6.6, o pico de força de tração medido na região occipital não deve exceder 4.170 N, para o valor de pico de compressão considera-se $4.000 \mathrm{~N}$, ambos os valores em qualquer tempo. O valor de momento máximo considerado foi de $\mathbf{5 7}$ N.m, conforme a antiga norma FMVSS 208.

Dependendo do plano que se avalia, no caso, "XZ", "YZ" e "XY", o movimento do pescoço terá quatro valores possíveis para $\mathrm{N}_{\mathrm{ij}}$, os quais consideram a atuação de efeitos combinados entre os esforços podendo ser: tração-extensão $\left(\mathrm{N}_{\mathrm{te}}\right)$; tração-flexão ( $\left.\mathrm{N}_{\mathrm{tf}}\right)$; compressão-extensão $\left(\mathrm{N}_{\mathrm{ce}}\right)$ e compressão-flexão $\left(\mathrm{N}_{\mathrm{cf}}\right)$. Ressalta-se que segundo a Fig. 9 (a), no plano "XZ" o pescoço pode se movimentar da direita para a esquerda; no plano "YZ" para frente e para trás e no plano "XY" a movimentação não é relevante, pois assume que a cabeça gira em 
torno de "Z". No caso do presente trabalho, consideram-se dois planos "XZ" e "YZ" cada um deles com os quatro efeitos possíveis, conforme será mostrado no item 3.1.

Segundo a norma, a cada tempo considerado apenas uma destas combinações pode acontecer, sendo as demais nulas. O valor da referida combinação é calculada conforme Eq. 2. Além disto, nenhuma destas combinações deve ser superior a um (1) a qualquer tempo da duração do evento.

A Tabela 3 resume os valores máximos associados a cada critério considerado seja para a cabeça, seja para o pescoço para um dummy Híbrido III 50\%, masculino.

Tabela 3 - Resumo dos valores máximos referentes aos critérios de lesão considerados

\begin{tabular}{l|l}
\hline Critério de Dano Cabeça & Valor máximo \\
\hline \hline $\mathrm{HIC} 15$ & 700 \\
\hline $\mathrm{A} 3 \mathrm{~ms}$ & $80 \mathrm{~g}$ \\
\hline Critério de Dano Pescoço & Valor máximo \\
\hline $\mathrm{N}_{\mathrm{ij}}\left(\mathrm{N}_{\mathrm{te}}, \mathrm{N}_{\mathrm{tf}}, \mathrm{N}_{\mathrm{ce}}, \mathrm{N}_{\mathrm{cf}}\right)$ & 1 \\
\hline $\mathrm{M}_{\max }$ & $57 \mathrm{~N} \cdot \mathrm{m}$ \\
\hline $\mathrm{F}_{\max }$ & $4.000 \mathrm{~N}(\mathrm{c}) \mathrm{e} 4.170 \mathrm{~N}(\mathrm{t})$ \\
\hline
\end{tabular}

\section{3 - SIMULAÇÃO DO TESTE ROOF CRUSH COM DUMMIES}

Este item traz os principais resultados relativos aos critérios de lesão de cabeça e pescoço apresentados no item 2 e resumidos na Tab. 3. Sendo assim, são consideradas em ambas as análises, a presença de quatro (4) dummies instrumentados, dois frontais e dois traseiros, que simulam a presença dos ocupantes de um veículo. A especificação destes é LS-Dyna Híbrido III 50\%, masculino com cintos de segurança.

O comportamento destes dummies, em caso de impacto, é analisado mediante a simulação numérica do teste experimental do Roof Crush, conforme apresentado no item 1.

\section{1 - Resultados da análise dos critérios de lesão - ocupantes do lado esquerdo}

Do estudo realizado, percebe-se que os ocupantes do lado esquerdo do veículo são mais penalizados que os do lado direito. Isto porque pelas características do Roofcrush o impacto é ressentido deste lado do veículo. Assim passageiros do lado direito têm, segundo os critérios de lesão utilizados (item 2), baixos valores de parâmetros de lesão de cabeça e pescoço. 

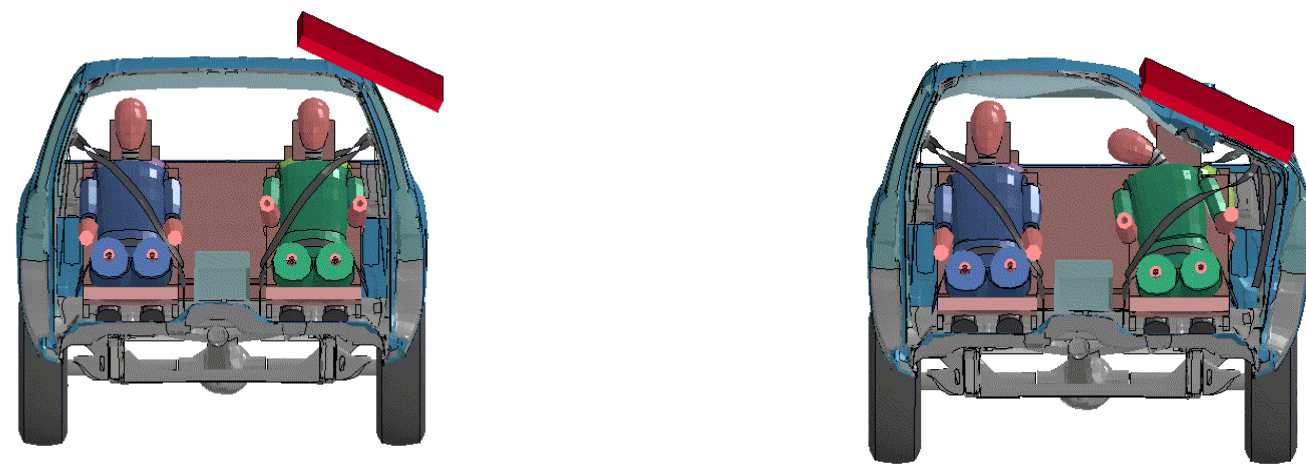

Figura 10 - Representação da simulação para estudo dos critérios de lesão: motorista e passageiro frontal $-\mathrm{t}=40$ $\mathrm{ms}$

A Figura 10 mostra a representação dos passageiros frontais com aplicação de força, conforme Roofcrush.

Os principais resultados obtidos para os ocupantes do lado esquerdo (motorista e passageiro traseiro atrás do motorista) são mostrados nas Figs. 11 e 12 e resumidos nas Tab. 4 e 5, respectivamente.

A Figura 11 traz alguns gráficos que da esquerda para a direita de cima para baixo ilustram: a aceleração da cabeça (em $\mathrm{m} / \mathrm{s}^{2}$ no gráfico mostrado em função de " $\mathrm{g}$ "); a força no pescoço (em kN); momento no pescoço (em N.m); intrusão (em mm); $\mathrm{N}_{\mathrm{ij}}$ plano XZ (adimensional) e $\mathrm{N}_{\mathrm{ij}}$ plano $\mathrm{YZ}$ (adimensional).

Percebe-se que no primeiro caso, $\mathrm{N}_{\mathrm{ij}}$ plano $\mathrm{XZ}$, entre parênteses, faz-se referência a (Fx, Fz e My) as forças são as que pertencem ao plano considerado e My momento perpendicular ao plano. Raciocínio análogo pode ser feito para $\mathrm{N}_{\mathrm{ij}}$ plano $\mathrm{YZ}$. O eixo ' $\mathrm{x}$ ' em todos os gráficos representa o tempo em $\mathrm{s}$.
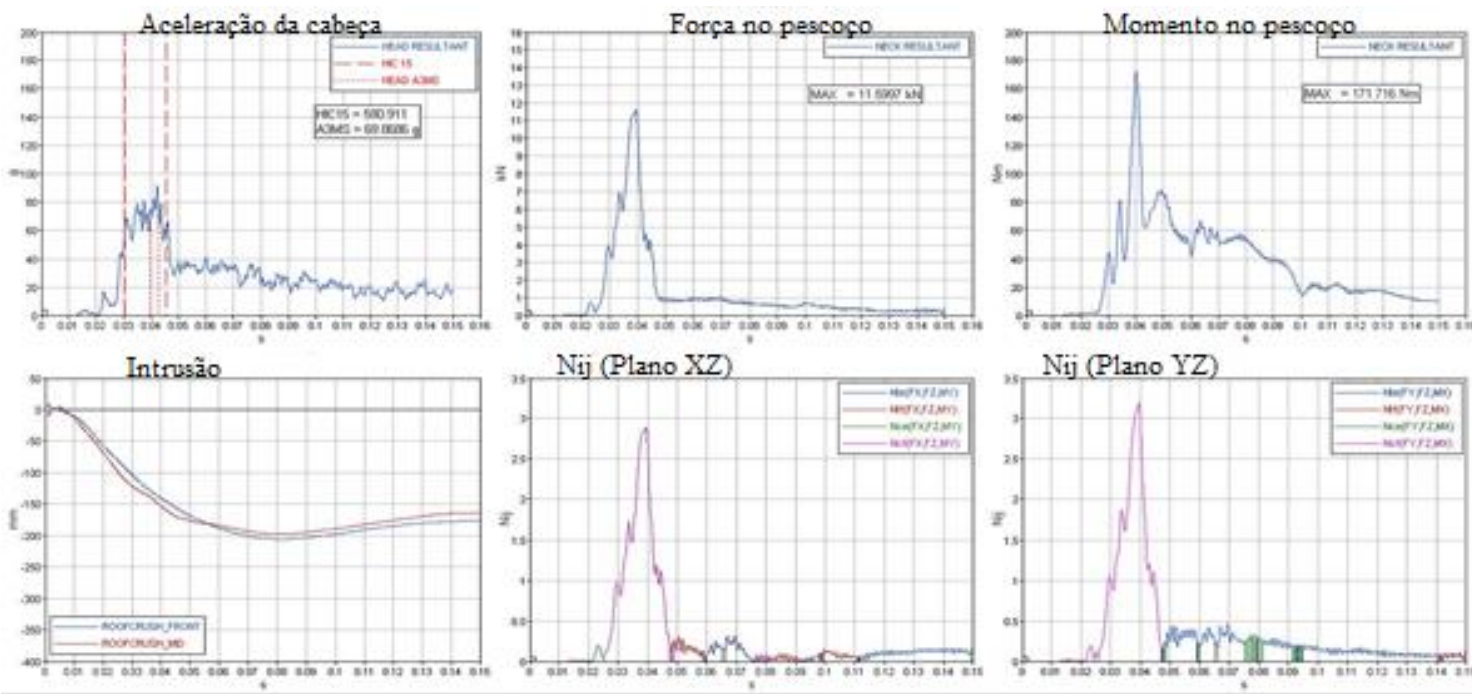

Figura 11 - Resultados dos critérios de lesão de cabeça e pescoço do motorista. 
No gráfico da aceleração da cabeça, cabem algumas observações. A curva azul mostra a resultante de aceleração no centro de gravidade da cabeça, no intervalo de tempo de 0 a $150 \mathrm{~ms}$.

As retas verticais vermelhas tracejadas mostram o intervalo de tempo em que o parâmetro de lesão da cabeça é calculado e é máximo, Eq. 1. Por exemplo, no primeiro gráfico da Fig. 11, tem-se que HIC15 é máximo com valor igual a 591 que acontece no intervalo $t_{1}=0,030$ s e $_{2}=0,04 \mathrm{~s}$. Estes valores serão os limites da integral da Eq. 1.

No caso de A3ms, retas pontilhadas vermelhas, o valor máximo da aceleração mantido durante $3 \mathrm{~ms}$ ocorre entre $\mathrm{t}=0,040 \mathrm{~s}$ e $\mathrm{t}=0,043 \mathrm{~s}$.

O gráfico referente à intrusão traz duas curvas que denotam a intrusão ocorrida nas colunas ' $A$ ' e ' $B$ ' do veículo. Repare que as curvas são bastante próximas no referido caso.

Os dois gráficos que se referem a $\mathrm{N}_{\mathrm{ij}}$ planos ' $\mathrm{XZ}$ ' e ' $\mathrm{YZ}$ ' tem ambos quatro (4) curvas. Estas representam os efeitos combinados dos esforços possíveis no pescoço, conforme tratado em 2.2.

A Tabela 4 abaixo resume os principais resultados. Percebe-se que os valores de força de compressão e momento máximo, assim como o $\mathrm{N}_{\mathrm{cf}}$ em ambos os planos, não atendem o estabelecido pelo regulamento FMVSS 208, Tab. 3.

\begin{tabular}{c|c} 
Tabela 4 - Principais resultados referentes aos crit \\
de lesão aplicado ao motorista \\
\hline $\mathrm{F}_{\max }$ compressão & $\mathbf{1 1 6 0 0 ~ \mathbf { ~ }}$ \\
\hline $\mathrm{M}_{\max }$ & $\mathbf{1 7 1 , 7}$ N.m \\
\hline HIC15 & $\mathbf{5 9 1}$ \\
\hline A3ms & $\mathbf{7 0} \mathbf{g}$ \\
\hline Nte (max plano XZ) & $\mathbf{0 , 3}$ \\
\hline Ntf (max plano XZ) & $\mathbf{0 , 3}$ \\
\hline Nce (max plano XZ) & $\mathbf{0 , 2}$ \\
\hline Ncf (max plano XZ) & $\mathbf{2 , 8}$ \\
\hline Nte (max plano YZ) & $\mathbf{0 , 4}$ \\
\hline Ntf (max plano YZ) & $\mathbf{0 , 1}$ \\
\hline Nce (max plano YZ) & $\mathbf{0 , 3}$ \\
\hline Ncf (max plano YZ) & $\mathbf{3 , 2}$ \\
\hline
\end{tabular}

Da Tab. 4, percebe-se que os valores marcados em vermelho suplantam os valores máximos estabelecidos na Tab. 3. O motorista sofre um forte impacto, por isso $F_{\max }$ 
e $\mathbf{M}_{\max }$ maiores que os valores referência, próxima a três vezes o valor padrão. Percebe-se que A3ms não ultrapassa o limite de $80 \mathrm{~g}$, mas é muito próximo.

A Figura 12 traz a mesma sequência de gráficos da Fig. 11 relativamente ao passageiro que se encontra atrás do motorista. As observações com relação ao formato das curvas também são os mesmos apresentados na Fig. 11.

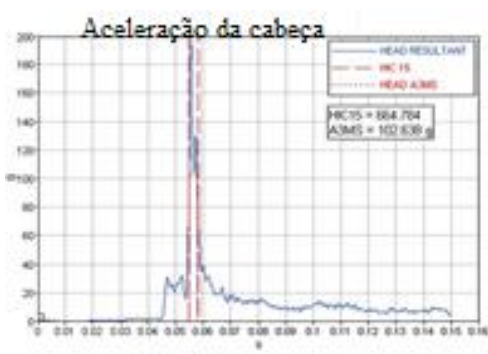

Intrusão
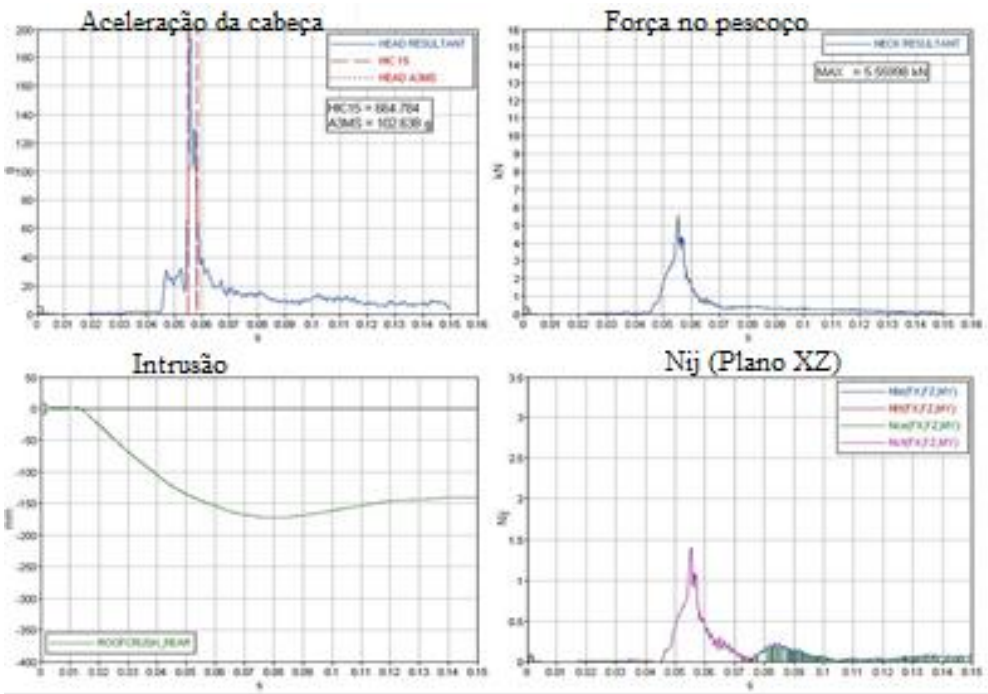

Nij (Plano XZ)

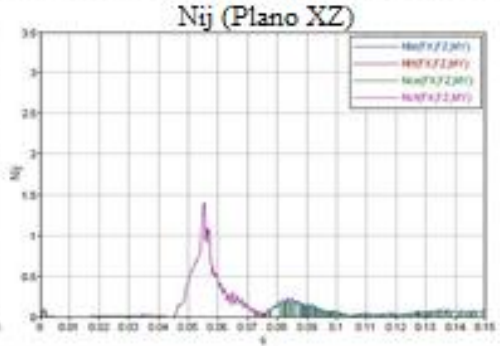

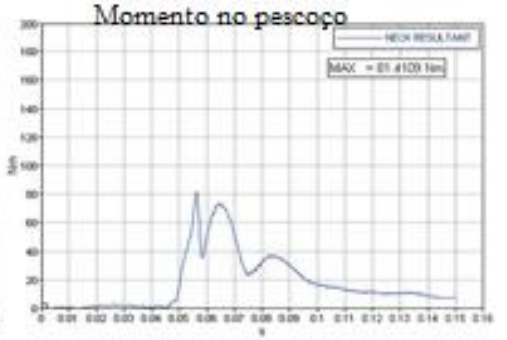

$\mathrm{Nij}$ (Plano YZ)

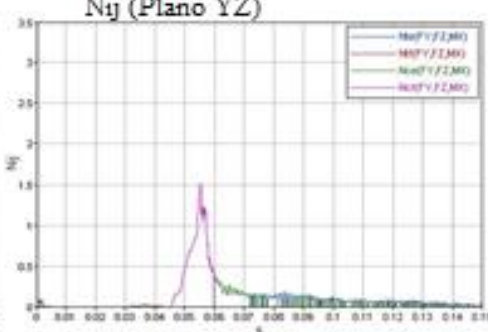

Figura 12 - Resultados dos critérios de lesão de cabeça e pescoço do ocupante atrás do motorista

A Tabela 5 traz os resultados obtidos da análise do gráfico da Fig. 12. Percebe-se que os valores de força de compressão, momento máximo, $\mathrm{N}_{\mathrm{cf}}$ (ambos os planos) e A3ms não atendem o estabelecido pelo regulamento FMVSS 208, Tab. 3.

Tabela 5 - Principais resultados referentes aos critérios de lesão do passageiro atrás do motorista

\begin{tabular}{|c|c|}
\hline $\mathrm{F}_{\max }$ compressão & $5560 \mathrm{~N}$ \\
\hline $\mathrm{M}_{\max }$ & 81 N.m \\
\hline $\mathrm{HIC} 15$ & 664,7 \\
\hline $\mathrm{A} 3 \mathrm{~ms}$ & $102,6 \mathrm{~g}$ \\
\hline Nte (max plano XZ) & 0,25 \\
\hline Ntf (max plano XZ) & $\mathbf{0 , 0 1}$ \\
\hline Nce (max plano XZ) & 0,25 \\
\hline Ncf (max plano XZ) & 1,4 \\
\hline Nte (max plano YZ) & 0,25 \\
\hline Ntf (max plano YZ) & $\mathbf{0 , 0 1}$ \\
\hline Nce (max plano YZ) & 0,20 \\
\hline Ncf (max plano YZ) & 1,5 \\
\hline
\end{tabular}


Como esperado, o ocupante atrás do motorista não atende os mesmos parâmetros indicativos de lesão do motorista. Da comparação dos resultados das Tabs. 4 e 5, percebe-se que a magnitude dos valores é menor que a do motorista, mas ainda elevados. Os resultados são coerentes, visto que em termos de intrusão os valores são da ordem de $200 \mathrm{~mm}$ nas colunas 'A' e 'B' e $175 \mathrm{~mm}$ na coluna ' $C$ '.

Além disso, dado o ângulo da placa do aparato experimental relativamente à superfície do veículo na parte voltada à traseira deste, Fig. 5, percebe-se que a movimentação do dummy frontal relativamente ao que está atrás é diferente. $\mathrm{O}$ valor de A3ms é menor no dummy frontal, Tab. 4 e 5, apesar do mesmo se deslocar mais para a direita, segundo visualização da simulação.

Da comparação dos resultados das Tabs. 4 e 5, cabe salientar que ao impacto da placa, o movimento sofrido pelo motorista e pelo o passageiro logo atrás dele são diferentes. Percebe-se que as acelerações do passageiro frontal são inferiores ao do detrás, em contrapartida os $\mathrm{N}_{\mathrm{ij}}$ são maiores, indicando que forças e momentos são mais significativos.

\section{2 - Resultados da análise dos critérios de lesão - ocupantes do lado direito}

Análise similar foi realizada para os passageiros do lado direito. No presente caso, percebe-se que todos os parâmetros de lesão são atendidos com margem de segurança importante. As Tabelas 6 e 7 trazem os resultados obtidos para estes dois ocupantes.

Tabela 6 - Principais resultados referentes aos critérios de lesão do passageiro ao lado do motorista

\begin{tabular}{c|c}
\hline$F_{\text {max }}$ compressão & $\mathbf{1 5 8} \mathbf{~}$ \\
\hline $\mathbf{M}_{\max }$ & $\mathbf{6} \mathbf{~ N m}$ \\
\hline HIC15 & $\mathbf{0 , 2 9}$ \\
\hline A3ms & $\mathbf{3 , 5} \mathbf{g}$ \\
\hline Nte (max plano XZ) & $\mathbf{0 , 0 1}$ \\
\hline Ntf (max plano XZ) & $\mathbf{0 , 0 1}$ \\
\hline Nce (max plano XZ) & $\mathbf{0 , 0 1}$ \\
\hline Ncf (max plano XZ) & $\mathbf{0 , 0 1}$ \\
\hline Nte (max plano YZ) & $\mathbf{0 , 0 1}$ \\
\hline Ntf (max plano YZ) & $\mathbf{0 , 0 1}$ \\
\hline Nce (max plano YZ) & $\mathbf{0 , 0 1}$ \\
\hline Ncf (max plano YZ) & $\mathbf{0 , 0 1}$ \\
\hline
\end{tabular}


Assim como nos ocupantes do lado esquerdo, o nível de aceleração A3ms é maior no passageiro da parte detrás. Entretanto, os resultados de força e momento do pescoço e HIC15 não são nulos, porque estes ocupantes se ressentem da deformação da carroceria do veículo e do deslocamento dos bancos após o impacto da placa do dispositivo até a fase final do contato. Em ambos os casos, Tab. 7 e 8 , os valores de $\mathrm{N}_{\mathrm{ij}}$ são muito baixos.

Tabela 7 - Principais resultados referentes aos critérios de lesão do passageiro atrás do ocupante ao lado do motorista

\begin{tabular}{c|c}
\hline$F_{\max }$ compressão & $\mathbf{2 5 0} \mathbf{~}$ \\
\hline $\mathbf{M}_{\max }$ & $\mathbf{1 0 , 4} \mathbf{~ N m}$ \\
\hline HIC15 & $\mathbf{0 , 2 4}$ \\
\hline A3ms & $\mathbf{4 , 1 8 ~ g}$ \\
\hline Nte (max plano XZ) & $\mathbf{0 , 0 1}$ \\
\hline Ntf (max plano XZ) & $\mathbf{0 , 0 1}$ \\
\hline Nce (max plano XZ) & $\mathbf{0 , 0 1}$ \\
\hline Ncf (max plano XZ) & $\mathbf{0 , 0 1}$ \\
\hline Nte (max plano YZ) & $\mathbf{0 , 0 1}$ \\
\hline Ntf (max plano YZ) & $\mathbf{0 , 0 1}$ \\
\hline Nce (max plano YZ) & $\mathbf{0 , 0 1}$ \\
\hline Ncf (max plano YZ) & $\mathbf{0 , 0 1}$ \\
\hline
\end{tabular}

\section{CONCLUSÃO}

De acordo com os resultados apresentados, percebe-se que alguns dos critérios de lesão apresentados pela norma FMVSS 208 são violados, no caso dos passageiros do lado esquerdo. Esta maior penalização era esperada visto que o carregamento, segundo o esquema experimental do Roofcrush, é aplicado deste lado.

Percebe-se, ainda, que os maiores critérios concentram-se na coluna "A", ou seja, aquela próxima ao motorista. Tal evidência se traduz nos resultados dos critérios referentes ao pescoço, onde $\mathrm{F}_{\text {máx }}$ de $11.600 \mathrm{~N}$ e $\mathrm{M}_{\text {máx }}$ de $171,1 \mathrm{~N}$.m foram determinados para o motorista e $\mathrm{F}_{\text {máx }}$ de $5.500 \mathrm{~N}$ e $\mathrm{M}_{\text {máx }} 81 \mathrm{~N}$.m para o passageiro logo atrás dele.

Relativamente às acelerações $\mathrm{A} 3 \mathrm{~ms}$ destes passageiros, pode-se inferir que no caso do passageiro atrás do motorista, segundo geometria do dispositivo a placa toca o veículo apenas na coluna "A", sendo assim no decorrer da simulação seu toque no veículo (coluna "B") provoca um nível de aceleração maior neste passageiro que no motorista (pequeno impacto). 
Como trabalho futuro, a expectativa da modelagem numérica com inserção do dispositivo de proteção externa, ROPS, é que se consiga reduzir os níveis de força, momento, aceleração nos passageiros do lado esquerdo e que os níveis de intrusão (deformação) nas colunas "A" e "B" sejam reduzidos. Isto porque a presença do dispositivo fornecerá ao teto do veículo rigidez e, este servirá de anteparo às intrusões ocasionadas pela rolagem. Trabalhos futuros também visam avaliar e comparar a eficiência dos ROPS internos quando comparados ao externo.

\section{AGRADECIMENTOS}

Os autores gostariam de agradecer à Mineradora VALE pelo financiamento do projeto, à FINATEC (Fundação de Empreendimentos Científicos e Tecnológicos) pelo trabalho de gestão de recursos e ao CTAG-IDIADA (The Galician Automotive Technology Centre) pela parceria no desenvolvimento das simulações numéricas.

\section{REFERÊNCIAS}

[1] Conroy, C. et al. Rollover crashes: Predicting serious injury based on occupant, vehicle and crash characteristics. Accident Analysis \& Prevention 38, pp. 835-842, 2006.

[2] Jiang, C., Neal-Sturgess, C. E. e Hu, Y.. Kinematics simulation and head injury analysis for rollovers using MADYMO. International Journal of Crashworthiness vol. 15, No. 5, pp. 505-515, 2010.

[3] Funk, J. R., Cormier, J. M., Manoogian, S. J. Comparison of risk factors for cervical spine, head, serious, and fatal injury in rollover crashes. Accident Analysis \& Prevention 45, pp. 6774, 2012.

[4] Mattos, G. A. et al. Head Injuries to Restrained Occupants in Single-Vehicle Pure Rollover Crashes. Traffic Injury Prevention 14, pp. 360-368, 2013.

[5] Parenteau, C. S. et al. Field relevance of a suite of rollover tests to real-world crashes and injuries. Accident Analysis \& Prevention, 35, pp. 103-110, 2003.

[6] Kerrigan, J. R et al. Test system, vehicle and occupant response repeatability evaluation in rollover crash tests: the deceleration rollover sled test. International Journal of Crashworthiness, vol. 16, nº 6, pp. 583-605, 2011.

[7] Stephenson, R. R. The case for a dynamic rollover test. International Journal of Crashworthiness, vol. 17, no 2 , pp. 119-124, 2012.

[8] Yan, L. et al. Comparison of vehicle kinematics and occupant responses between Jordan rollover system and an over-the-load rollover. International Journal of Crashworthiness, vol. 17, No. 2, pp. 173-194, 2012.

[9] NASS - CDS. Coding manual, 1996.

[10] Department of Transportation (DOT), National Highway Traffic Safety Administration, Federal Motor Vehicle Safety Standards, Part 571 No 216, "Roof crush resistance" (2008). Available at http://www.fmcsa.dot.gov/rulesregulations/administration/fmcsr/fmcsrruletext.aspx?reg=571.216. 